\title{
Drug Interaction of Glycyrrhiza glabra L. with Chemical Drugs: A mini Review study
}

\author{
Mahmoud Bahmani', Pegah Shakib ${ }^{2 *}$, Pouya Parsaei ${ }^{3,4}$ \\ 'Biotechnology and Medicinal Plants Research Center, Ilam University of Medical Sciences, Ilam, Iran \\ ${ }^{2}$ Razi Herbal Medicines Research Center, Lorestan University of Medical Sciences, Khorramabad, Iran \\ ${ }^{3}$ Department of Food Hygiene, Faculty of Veterinary Medicine, Shahrekord Branch, Islamic Azad University, Shahrekord, Iran \\ ${ }^{4}$ Research Center of Nutrition and Organic Products, Shahrekord Branch, Islamic Azad University, Shahrekord, Iran
}

\section{*Correspondence to: \\ shakib.pegah@yahoo.com}

Received: 7 June 2020

Accepted: 19 June 2020

ePublished: 21 Novamber 2020

Keywords: Medicinal plant, Glycyrrhiza glabra L., Drug interaction

\section{Citation:}

Bahmani M, Shakib $\mathrm{P}$, Parsaei P. Drug Interaction of of the Fabaceae family that is a valuable food Glycyrrhiza glabra L. with and medicinal plant [1]. G. glabra L. as a weed Chemical Drugs: A mini Re- in wheat, summer and weed fields, cotton, poview Article. Plant Biotech- tatoes, sugar beet and fodder including alfalfa, nol Persa 2020; 2(2): 28-30. sainfoin and clover and due to high root and rhizome development reduces yields in fields and orchards $[2,3]$. The height of G. glabra L. varies and its height is between 100 to $200 \mathrm{~cm}$. G. glabra $\mathrm{L}$. has dense foliage.

The leaves are compound and have four to seven pairs of leaflets and one terminal leaflet. The flowers are irregular and appear yellow, purple or purple. The fruit is two to three centimeters long and brown in color. The sides of the fruit are narrow and more or less pointed. The seed coat is thick and strong and weighs about ten grams [4-6]. Distribution G. glabra L. is cultivated in Italy, Greece, England, Belgium, France, Turkey, Iran and Germany $[7,8]$.

In traditional medicine, it is used to treat duodenal ulcers, coughs, sore throats, relieve inflammation, bloating, strong laxatives and antidotes, hot flashes, relieve thirst, relieve skin discomfort and anti-allergies [9-11]. The most important active ingredient in this plant is glycyrrhizic acid [12]. Other chemical constituents of G. glabra L. include liquiritin, isoliquiritin, liquiritigenin, isoliquiritigenin, 22, 23-dihy- droastigmastrol, asparaginase, glabridin, galbrene and licochalcone A, B, C, D [13-17].

Glycyrrhizic acid with the chemical formula $\mathrm{C}_{42} \mathrm{H}_{62} \mathrm{O}_{16}$ is composed of one molecule of glycerotenic acid and two molecules of glucuronic acid $[18,19]$. G. glabra L. is a widely used plant in Iran and medicinal products have been produced from it in Iran. Medicinal products produced from G. glabra L. plant available in the Iranian market include lycopene tablets that are anti-inflammatory, expectorant and used as an anti-cough. Metazine tablets are used for purposes such as improving gastrointestinal pain, treating stomach ulcers, and anti-flatulence and laxatives. Reglis tablets that are prescribed to treat bloating and duodenum, excessive acid secretion and bloating. D-Reglis tablets are also used to prevent peptic ulcer in concomitant use with nonsteroidal anti-inflammatory drugs.

drug interactions [20]. Licorice syrup for the treatment of gastric, duodenal ulcers, gastritis and gastrology; Reglycidine tablets are used to treat bloating, duodenum, gastritis and gastrology, and altladin tablets are used to treat inflammation and irritation of the laryngeal mucosa, sputum in irritating coughs. Gastrin tablets are also produced and prescribed as anti-inflammatory and analgesic for stomachpain, accelerating the healing of gastritis and duodenal ulcers. Prescribing several drugs to

Copyright (C) 2020 The Author(s). This is an open-access article distributed under the terms of the Creative Commons Attribution License (http://creativecommons.org/licenses/by/4.0), which permits unrestricted use, distribution, and reproduction in any medium, provided the original work is properly cited. 
treat diseases is common and unavoidable and sometimes causes drug interactions [20].

Interactions between herbal and chemical drugs are also common. In this review study, drug interactions between $G$. glabra L. and chemical drugs were investigated and reported. $G$. glabra L. inhibits the metabolism and pharmacokinetics of prednisolone [21-24]. Studies show that G. glabra L. intensifies the effect of glucocorticoids [25-27]. The results of a study showed that glycerinic acid of the plant intensifies the cutaneous vasoconstrictive response of hydrocortisone. In another study, glycyrrhizin (Glycyrrhiza glabra) decreased the plasma clearance of prednisolone and increased the plasma concentration of prednisolone [28]. This herbal medicine can increase blood pressure by retaining sodium and fluids [29]. G. glabra L. exacerbates hypokalemia [30] and reduces the effect of spironolactone [27]. G. glabra L. interferes with antihypertensives and raises blood pressure through sodium and fluid retention and hypokalemia [29]. The drug interaction of this plant with laxatives is due to increased potassium excretion, which has side effects such as weakness, arrhythmia and confusion [31]. Drug Interaction $G$. glabra L. with antibiotics, plant activity is reduced by drug inhibition of intestinal microbial flora [21].

Among the drug interactions of G. glabra L. with Hypoglycemics, it can be mentioned that the plant can reduce glucose tolerance [29]. Drug interaction of this plant with Aspirin is a reduction in drug-induced gastric bleeding [21]. G. glabra L. also interacts with Warfarin, so the plant may reduce platelet activity [29]. Hypokalemia, hypertension, and edema are drug interactions of G. glabra L. with OCPs [24]. Drug interactions of G. glabra L. with Digitalis glycosides and digoxin include an increased risk of digitalis toxicity, hypokalemia, and pharmacodynamic interactions and drug levels [32].

\section{Conclusion}

The results of this short review study showed that the drug G. glabra L. can interact with various chemical drugs, so the concomitant use of G. glabra L. with other drugs requires caution.

\section{Authors' contribution}

All authors contributed equally to the manuscript.

\section{Conflicts of interest}

The authors declared no competing interests.

\section{Ethical considerations}

Ethical issues (including plagiarism, data fabrica $\neg$ tion, double publication and etc.) have been completely ob $\neg$ served by author.

\section{Funding/Support None.}

\section{References}

1. Amani M, Sotudeh-Gharebagh R, Mostaoufi N, Kashani H. Optimal Exteraction of Glycyrrhetinic Acid From Licorice Root. J Technol. 2005; 3 (4):376-580.

2. Karimielise $\mathrm{H}$. Information about licorice. Forest and Range. 1989; 6: 8-12.

3. Chandler F. Herbs Everyday Refrence for Healt professionals. Canadian Pharmacists Association and the Canadian Medicinal Association. 2000.

4. Akhundzadeh S. Encyclopedia of Iranian Medicinal plants.
Institue of Medicinal Plants. Jahad-e Daneshgahi. 2000; 213.

5. Ghahraman A. Basic Botany: Anatomy and Morphology, Vol. 1. University of Tehran Press. 1999: 539.

6. Omidbeigi R. Processing and production of medicinal plants. Razavi Publications, Mashhad. 2006; 3: 397.

7. AOAC. Association of Official Analytical Chemists, Official method 982.19. 2002.

8. Mirhaidar H. Licorice, Herbal plants used in the treatment of diseases and education. Office of Islamic culture publication. 1994; 3: 6-12.

9. Bluemental M. Herbal medicine expanded comission emonographs. 1st ed. United States of America: Integrative Medicine Communication; 2000.

10. Mirheidar $\mathrm{H}$. Botany application of plants in prevention and treatment of diseases. 4th ed. Tehran: Daftar Nashr-e-Farhang Islamic; 2003; 3: 1-20.

11. Chopin lucks B. Vitex agnus castus essential oil and menopausal balance: a research update. Complement Ther Nurs Midw. 2003; 9(3): 157-60.

12. Tyler VE, Bradly LR, Robbers JE. Pharmacognosy. 9th ed. Lea and Febiger. Philadlphia. 1988: 68-69.

13. Tyler VE, Bradly LR, Robbers JE. Pharmacognosy. 9th ed. Lea and Febiger. Philadlphia. 1988: 68-69.

14. Blumenthal M, Goldberg A, Brinckmann J. Herbal Medicine: Expanded Commission E Monographs. Newton. American Botanical Council. 2000: 233-236.

15. Hayashi H, Hiraoka N, Ikeshiro Y, Yamamoto H, Yoshikawa T. Seasonal variation of glycyrrhizin and isoliquiritigenin glycosides in the root of Glycyrrhiza glabra L. Biol Pharm Bull. 1998; 21: 987-989.

16. De Simone F, Aquino R, De Tommasi N, Mahmood N, Piacente S, Pizza C: Anti-HIV aromatic compounds from higher plants. In: Tringali C, (ed). Bioactive Compounds from Natural Sources: Isolation, Characterization and Biological Properties. Taylor and Francis Inc. New York. 2001: 325.

17. Haraguchi H. Antioxidative plant constituents. In: Tringali, C, (ed). Bioactive Compounds from Natural Sources: Isolation, Characterization and Biological Properties. Taylor and Francis Inc. New York. 2001: 348-352.

18. Mehravar M. Extraction of licorice from licorice roots by pure water and one percent aqueous ammonia solution. M.Sc. Thesis. Shiraz University, Shiraz, Iran. 1991: 107.

19. Marzi V, Circella G and Vampa GM. Effect of soil depth on the rooting system growth in Glycyrrhiza glabra L. ISHS Acta Horticulture 1993; 331: 71-78.

20. Beijnen JH, Schellens JH. Drug interactions in oncology. Lancet Oncol2004; 5(8): 489-496.

21. http://www.vitalgoods.com/myibis/herbdru g.pdf.

22. Vickers A, Zollman CA. ABC of Complementary medicine, Herbal medicine. BMJ 1999; 319: 1050-58.

23. http://www.bodyandfitness.com/bodyandfit ness1.html.

24. Shih RD. Rosens Emergency Medicine: Concepts and Clinical Practice, 5th ed, Mosby, Inc. 2002: 2204.

25. Kuhn MA. Herbal Remedies: Drug - Herb Interactions. Critical Care Nurse. 2002; 22: 22- 32.

26. Fleming T. PDR for Herbal Medicine. Second Edition; Medical economics Co. Montvale New Jersey, 2000.

27. Corponter DO. Nursing herbal medicine Handbook. Springhouse corporation, springhouse. Pennsylvania; 2001: 483488.

28. Fugh-Berman A. Herb and drug interaction. Lancet 2000; 355: 134-8.

29. Brent J. Herbal drug interaction chart. http:// www.sdh. sk.ca/rxfiles. July 2002. 
30. Mills S and Bone K. Principle and practice of phytotherapy (Modern herbal medicine). Churchill Livingstone, 2000: 104-7.

31. http://www.vitalgoods.com/myibis/herbdru g.pdf.

32. Vickers A, Zollman CA. ABC of Complementary medicine, Herbal medicine. BMJ 1999; 319: 1050-58. 